DOI: $10.15393 /$ j3.art.2016.3350

UDC 517.165

\author{
F. M. Dannan, S. M. Sitnik
}

\title{
THE DAMASCUS INEQUALITY
}

\begin{abstract}
In 2016 Prof. Fozi M. Dannan from Damascus, Syria, proposed an interesting inequality for three positive numbers with unit product. It became widely known but was not proved yet in spite of elementary formulation. In this paper we prove this inequality together with similar ones, its proof occurred to be rather complicated. We propose some proofs based on different ideas: Lagrange multipliers method, geometrical considerations, Klamkin-type inequalities for symmetric functions, usage of symmetric reduction functions of computer packages. Also some corollaries and generalizations are considered, they include cycle inequalities, triangle geometric inequalities, inequalities for arbitrary number of values and special forms of restrictions on numbers, applications to cubic equations and symmetric functions.
\end{abstract}

Key words: cycle inequalities, Lagrange method, geometric inequalities, symmetric reduction

2010 Mathematical Subject Classification: 26D15

1. The problem formulation. In 2016 Prof. Fozi M. Dannan from Damascus, Syria, proposed the following inequality

$$
\frac{x-1}{x^{2}-x+1}+\frac{y-1}{y^{2}-y+1}+\frac{z-1}{z^{2}-z+1} \leq 0,
$$

provided that $x y z=1$ for $x, y, z>0$. It became widely known but was not proved yet in spite of elementary formulation.

An obvious generalization is the next inequality

$$
\sum_{k=1}^{n} \frac{x_{k}-1}{x_{k}^{2}-x_{k}+1} \leq 0
$$


providing that $x_{1} \cdot x_{2} \cdot \ldots \cdot x_{n}=1$ for $x_{k}>0,1 \leq k \leq n$.

It is obvious that (2) is true for $n=1$, it is easy to prove it also for $n=2$ directly. But it is not true for $n=4$ as follows from an example with $x_{1}=x_{2}=x_{3}=2, x_{4}=\frac{1}{8}$, then (2) is reduced to $1-\frac{56}{57} \leq 0$ which is not true.

As a consequence $(2)$ is also false for any $n \geq 4$ due to an example with $x_{1}=x_{2}=x_{3}=2, x_{4}=\frac{1}{8}, x_{5}=\ldots=x_{n}=1$. So the only non-trivial case in (2) is $n=3$.

In this paper we prove inequality (1) together with similiar ones

$$
\begin{aligned}
& \frac{1}{x^{2}-x+1}+\frac{1}{y^{2}-y+1}+\frac{1}{z^{2}-z+1} \leq 3, \\
& \frac{x}{x^{2}-x+1}+\frac{y}{y^{2}-y+1}+\frac{z}{z^{2}-z+1} \leq 3, \\
& \frac{x-1}{x^{2}+x+1}+\frac{y-1}{y^{2}+y+1}+\frac{z-1}{z^{2}+z+1} \leq 0, \\
& \frac{1}{x^{2}+x+1}+\frac{1}{y^{2}+y+1}+\frac{1}{z^{2}+z+1} \geq 1, \\
& \frac{x}{x^{2}+x+1}+\frac{y}{y^{2}+y+1}+\frac{z}{z^{2}+z+1} \leq 1, \\
& \frac{x+1}{x^{2}+x+1}+\frac{y+1}{y^{2}+y+1}+\frac{z+1}{z^{2}+z+1} \leq 2 .
\end{aligned}
$$

Also some generalizations and applications will be considered.

\section{Proof of the main inequality (1).}

Theorem 1. Inequality (1) is true providing that $x y z=1$ for $x, y, z>0$.

For the proof we need an auxiliary inequality that seems to be very interesting by itself.

Lemma 1. Let $x, y, z$ be positive numbers such that $x y z=1$. Then

$$
x^{2}+y^{2}+z^{2}-3(x+y+z)+6 \geq 0
$$

is true.

Note that inequality $(9)$ is not a consequence of well-known family of Klamkin-type inequalities for symmetric functions [1]. So (9) is a new quadratic Klamkin-type inequality in three variables under restriction 
$x y z=1$. Due to its importance we give three proofs to it based on different ideas.

First proof of Lemma 1. To prove (9) introduce the Lagrange function

$$
L(x, y, z, \lambda)=x^{2}+y^{2}+z^{2}-3(x+y+z)+6-\lambda(x y z-1) .
$$

Differentiating it get

$$
\lambda=2 x^{2}-3 x=2 y^{2}-3 y=2 z^{2}-3 z .
$$

It follows that at the minimum (it obviously exists) $x=y$ or $x+y=1.5$.

For the first case three variables at the minimum are $x, y=x, z=$ $=1 / x^{2}$. From $2 x^{2}-3 x=2 z^{2}-3 z$ we derive the equation for $x$ :

$$
2 x^{2}-3 x=2 / x^{4}-3 / x^{2}, \quad f(x)=2 x^{6}-3 x^{5}+3 x^{2}-2=0 .
$$

One root is obvious $x=1$. Let us prove that there are no other roots for $x \geq 0$. Check that derivative is positive

$$
f^{\prime}(x)=12 x^{5}-15 x^{4}+6 x=3 x\left(4 x^{4}-5 x^{3}+2\right) \geq 0, x \geq 0 .
$$

Define a function $g(x)=4 x^{4}-5 x^{3}+2$, its derivative $g^{\prime}(x)$ has one zero for $x \geq 0$ at $x=15 / 16$ and the function $g(x)$ is positive in this zero (its minimum) $g(15 / 16)=15893 / 16384>0$. So $g(x)$ is positive, $f(x)$ is strictly increasing on $x \geq 0$, so $f(1)=0$ is its only zero.

For the second case $x+y=1.5$ the proof is the same as below (case II of the second proof of Lemma 1).

Second proof of Lemma 1. Consider the function

$$
f(x, y)=x^{2}+y^{2}+\frac{1}{x^{2} y^{2}}-3\left(x+y+\frac{1}{x y}\right)+6,
$$

where $x, y, z$ are positive numbers. We show that $f(x, y)$ attains its minimum 0 at $x=1, y=1$ using partial derivative test.

Calculate

$$
\begin{aligned}
& \frac{\partial f}{\partial x}=2 x-\frac{2}{x^{3} y^{2}}-3+\frac{3}{x^{2} y}=0, \\
& \frac{\partial f}{\partial y}=2 y-\frac{2}{x^{2} y^{3}}-3+\frac{3}{x y^{2}}=0 .
\end{aligned}
$$

Now multiplying (10) and (11) respectively by $x$ and $-y$ and adding obtain

$$
(x-y)(2 x+2 y-3)=0 .
$$


Here we have two cases.

Case I. $x=y$, which implies from equation t10 that

$$
2 x^{6}-2-3 x^{5}+3 x^{2}=0
$$

or

$$
\left(x^{3}-1\right)\left(2 x^{3}-3 x^{2}+2\right)=0 .
$$

Equation 12 has only one positive root $x=1$ and consequently $y=1$. Notice that the equation

$$
2 x^{3}-3 x^{2}+2=0
$$

does not have positive roots because for $x \geq 0$ the function $u(x)=2 x^{3}-$ $-3 x^{2}+2$ satisfies the following properties: $(i) u(0)=2,(i i) \min u(x)=1$ at $x=1,($ iii $) u(\infty)=\infty$. Therefore $f(x, y)$ attains its maximum or minimum at $x=1, y=1$.

Case II. $2 x+2 y=3$. Adding (10) and (11) we get

$$
2(x+y)-6-2\left(\frac{1}{x^{3} y^{2}}+\frac{1}{x^{2} y^{3}}\right)+3\left(\frac{1}{x^{2} y}+\frac{1}{x y^{2}}\right)=0
$$

or

$$
-3-\frac{3}{x^{3} y^{3}}+\frac{3}{2 x^{2} y^{2}}=0
$$

and

$$
-6 x^{3} y^{3}+3 x y-6=0 .
$$

Putting $t=x y$ we obtain

$$
2 t^{3}-t+2=0
$$

In fact this equation does not have positive roots (notice that $t=x y$ should be positive). This is because the function $u=2 t^{3}-t+2$ satisfies the following properties:

$$
\text { (i) } u(0)=2 \text {, }
$$$$
\text { (ii) for } t>0, \min u(t)=u\left(\frac{1}{\sqrt{6}}\right)>0,(\text { iii }) u(\infty)>0 \text {. }
$$

The last step is to show that

$$
f(x, y) \geq f(1,1)=0
$$


It is enough to show that $f(s, t)>f(1,1)$ for at least one point $(s, t) \neq$ $\neq(1,1)$. Take for example $f(2,3)=\frac{7}{2}+\frac{1}{36}$.

Third proof of Lemma 1 (Geometrical Method). Geometrically it is enough to prove that the surface $x y z=1$ lies outside the sphere $(x-3 / 2)^{2}+(y-3 / 2)^{2}+(z-3 / 2)^{2}=3 / 4$ except the only intersection point $(1,1,1)$ as it is shown in the next graph:

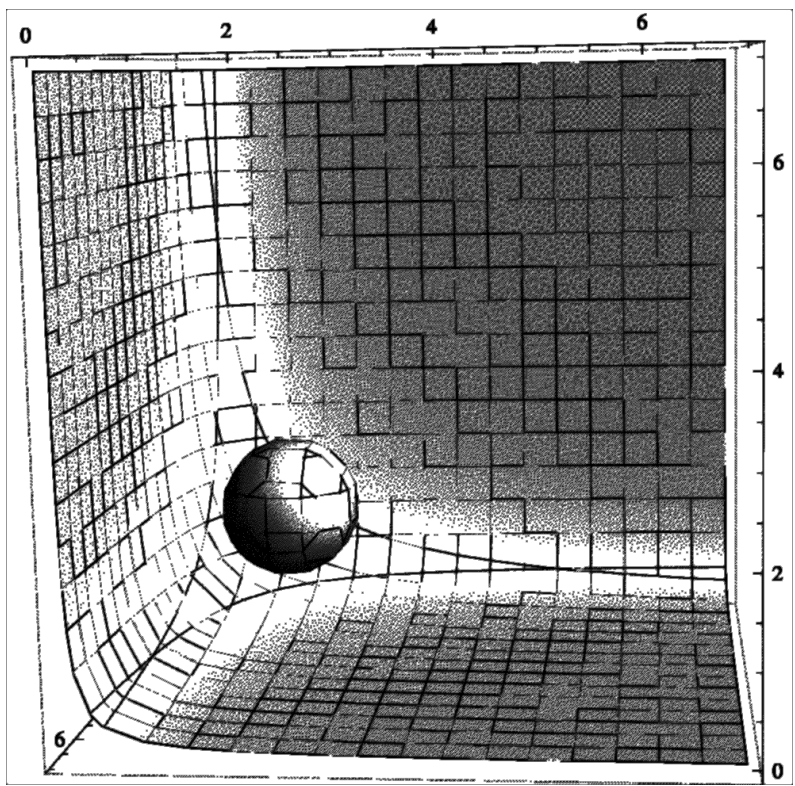

Figure 1: the sphere on the surface.

Let $M$ and $S$ be surfaces defined by

$M: x y z=1$ and $S:\left(x-\frac{3}{2}\right)^{2}+\left(y-\frac{3}{2}\right)^{2}+\left(z-\frac{3}{2}\right)^{2}-\frac{3}{4}=0$.

1. If $\left(z-\frac{3}{2}\right)^{2}-\frac{3}{4} \geq 0$ then

$$
\left(x-\frac{3}{2}\right)^{2}+\left(y-\frac{3}{2}\right)^{2}+\left(z-\frac{3}{2}\right)^{2}-\frac{3}{4} \geq 0
$$

and equivalently

$$
x^{2}+y^{2}+z^{2}-3(x+y+z)+6 \geq 0 .
$$

2. If $\left(z-\frac{3}{2}\right)^{2}-\frac{3}{4} \leq 0$ then $\frac{3-\sqrt{3}}{2} \leq z \leq \frac{3+\sqrt{3}}{2}$.

3. We take horizontal sections for both $M$ and $S$ so get for any plane

$$
\frac{3-\sqrt{3}}{2} \leq z=k \leq \frac{3+\sqrt{3}}{2}
$$


two curves: equilateral hyperbola $H(k)$ with vertex $\left(\frac{1}{\sqrt{k}}, \frac{1}{\sqrt{k}}\right)$ and a circle $C(k)$ with center at $\left(\frac{3}{2}, \frac{3}{2}, k\right)$ and radius given by

$$
r^{2}(k)=\frac{3}{4}-\left(k-\frac{3}{2}\right)^{2}=-k^{2}+3 k-\frac{3}{2} .
$$

4. For $z=1$, we have the hyperbola $x y=1$ and the circle

$$
\left(x-\frac{3}{2}\right)^{2}+\left(y-\frac{3}{2}\right)^{2}=\frac{1}{2} .
$$

5 . We show that the distance $d(v, c)$ between the vertex of the hyperbola and the center $c$ of the circle is always greater than or equal to the radius of the circle. The distance $d(v, c)$ is given by

$$
d^{2}(v, c)=\left(\frac{1}{\sqrt{k}}-\frac{3}{2}\right)^{2}+\left(\frac{1}{\sqrt{k}}-\frac{3}{2}\right)^{2}=2\left(\frac{1}{\sqrt{k}}-\frac{3}{2}\right)^{2} .
$$

The radius is given by

$$
r^{2}(k)=\frac{3}{4}-\left(k-\frac{3}{2}\right)^{2}=-k^{2}+3 k-\frac{3}{2} .
$$

We need to show that the vertex is always outside the circle i.e. $d^{2}(v, c) \geq$ $\geq r^{2}(k)$ for all

$$
\frac{3-\sqrt{3}}{2} \leq k \leq \frac{3+\sqrt{3}}{2} .
$$

It is clear that $d(v, c)=r$ for $k=1$ and the hyperbola tangents the circle at the point $(1,1,1)$.

For $\frac{3-\sqrt{3}}{2} \leq k<1$, as $k$ decreases from 1 to $\frac{3-\sqrt{3}}{2}$, the radius of the circle becomes smaller. From the other side the vertex $\left(\frac{1}{\sqrt{k}}, \frac{1}{\sqrt{k}}, k\right)$ moves away from $(1,1,1)$ towards the point $(0,0, k)$. This follows from the distance function of the vertex

$$
O v=\frac{\sqrt{2}}{\sqrt{k}},\left(0<k_{1} \leq k_{2}<1 \rightarrow \frac{\sqrt{2}}{\sqrt{k_{2}}}<\frac{\sqrt{2}}{\sqrt{k_{1}}}\right) .
$$

6. For $1<k \leq \frac{3+\sqrt{3}}{2}$, we show that

$$
d^{2}(v, c)=g(k)=2\left(\frac{1}{\sqrt{k}}-\frac{3}{2}\right)^{2}>r^{2}=\frac{3}{4}-\left(k-\frac{3}{2}\right)^{2}=h(k) .
$$


In fact, $h(k)$ is a concave down parabola and has its maximum at $k=1$, i.e. $\max h(k)=\frac{1}{2}$ and $h(k)$ is decreasing for $k>1$. Also, $g(1)=\frac{1}{2}$ and $g(k)$ is increasing for $k>1$ because $g^{\prime}(k)=4\left(-\frac{1}{k \sqrt{k}}\right)\left(\frac{1}{\sqrt{k}}-\frac{3}{2}\right)>0$ for $k \geq 1$ (notice that $\frac{1}{\sqrt{k}}<1$ ). Therefore $g(k)>h(k)$ for $k>1$. Eventually we conclude that

$$
\left(x-\frac{3}{2}\right)^{2}+\left(y-\frac{3}{2}\right)^{2}+\left(z-\frac{3}{2}\right)^{2}-\frac{3}{4} \geq 0
$$

and consequently

$$
x^{2}+y^{2}+z^{2}-3(x+y+z)+6 \geq 0
$$

for all values of $(x, y, z)$ that satisfy $x y z=1$.

Proof of Theorem 1. Now consider the inequality to prove (1). After simplifying inequality (1) with the use of Wolfram Mathematica, we get

$$
\begin{array}{r}
-3+3 x-2 x^{2}+3 y-3 x y+2 x^{2} y-2 y^{2}+2 x y^{2}-x^{2} y^{2}+3 z-3 x z+ \\
+2 x^{2} z-3 y z+3 x y z-2 x^{2} y z++2 y^{2} z-2 x y^{2} z+x^{2} y^{2} z-2 z^{2}+2 x z^{2}- \\
-x^{2} z^{2}+2 y z^{2}-2 x y z^{2}+x^{2} y z^{2}-y^{2} z^{2}+x y^{2} z^{2} \leq 0 .
\end{array}
$$

Using Symmetric Reduction function of Wolfram Mathematica we derive

$$
\begin{array}{r}
3-x y-x z-y z+3 x y z-3(x+y+z)+2(x+y+z)^{2}- \\
-x y z(x y+x z+y z)-2(x+y+z)(x y+x z+y z)+(x y+x z+y z)^{2} \geq 0 .
\end{array}
$$

Using $x y z=1$, the required inequality becomes

$$
\begin{array}{r}
6-3(x+y+z)+2(x+y+z)^{2}-2(x y+x z+y z)- \\
-2(x+y+z)(x y+x z+y z)+(x y+x z+y z)^{2} \geq 0 .
\end{array}
$$

In terms of elementary symmetric functions

$$
S_{1}=x+y+z, S_{2}=x y+y z+x z
$$

it is

$$
S_{2}^{2}-2 S_{1} S_{2}-2 S_{2}+2 S_{1}^{2}-3 S_{1}+6 \geq 0 .
$$

As $S_{2}^{2}-2 S_{1} S_{2}+S_{1}^{2} \geq 0$, it is enough to prove

$$
S_{1}^{2}-2 S_{2}-3 S_{1}+6 \geq 0 .
$$


Inequality 14 is equivalent to

$$
x^{2}+y^{2}+z^{2}-3(x+y+z)+6 \geq 0,
$$

which is true from Lemma 1 . So Theorem 1 is proved.

3. Proof of inequalities (3)-(8). Let us start with two propositions. Proposition 1. For any real numbers $u, v, w$ such that

$$
(1+u)(1+v)(1+w)>0
$$

the inequality

$$
\frac{1}{1+u}+\frac{1}{1+v}+\frac{1}{1+w} \leq k(\geq k)
$$

is equivalent to

$$
k u v w+(k-1)(u v+v w+w u)+(k-2)(u+v+w)+k-3 \geq 0(\leq 0) .
$$

Proposition 2. For any real numbers $u, v, w$ such that

$$
(u-1)(v-1)(w-1)>0
$$

the inequality

$$
\frac{1}{u-1}+\frac{1}{v-1}+\frac{1}{w-1} \leq k(\geq k)
$$

is equivalent to

$k u v w-(k+1)(u v+v w+w u)+(k+2)(u+v+w)-(k+3) \geq 0(\leq 0)$.

The validity of propositions 1 and 2 can be obtained by direct expansions.

Proof that $(1) \Leftrightarrow(3)$. In fact

$$
\begin{gathered}
\frac{x-1}{x^{2}-x+1}+\frac{y-1}{y^{2}-y+1}+\frac{z-1}{z^{2}-z+1}= \\
=\frac{x^{2}-\left(x^{2}-x+1\right)}{x^{2}-x+1}+\frac{y^{2}-\left(y^{2}-y+1\right)}{y^{2}-y+1}+\frac{z^{2}-\left(z^{2}-z+1\right)}{z^{2}-z+1}= \\
=-3+\sum_{c y c} \frac{x^{2}}{x^{2}-x+1} .
\end{gathered}
$$


Now if the right-hand side is less than or equal to zero then

$$
\sum_{c y c} \frac{x^{2}}{x^{2}-x+1} \leq 3
$$

and consequently

$$
\sum_{c y c} \frac{1}{1-(1 / x)+(1 / x)^{2}} \leq 3 .
$$

Proof of (5). We need to prove

$$
\sum_{c y c} \frac{1}{x^{2}+x+1} \geq 1 \text {. }
$$

Let $u=x^{2}+x, v=y^{2}+y, w=z^{2}+z$. Using Proposition 1 the required inequality can be written as follows:

$$
u v w-(u+v+w)-2 \leq 0 .
$$

Going back to $x, y, z$ we get

$$
(x+1)(y+1)(z+1)-\left(x^{2}+y^{2}+z^{2}\right)-(x+y+z)-2 \leq 0 .
$$

Or

$$
x y+y z+z x \leq x^{2}+y^{2}+z^{2}
$$

which is obvious.

Proof of (6). It follows from elementary calculus that for any real number $x$ we have

$$
\frac{x}{x^{2}+x+1} \leq \frac{1}{3}
$$

and the inequality follows directly.

Proof that $(6)+(7) \Rightarrow(5)$. Adding together (7) and (6) multiplied by -1 we derive (5).

Proof that $(6) \Rightarrow(8)$. The required inequality is equivalent to

$$
\sum_{c y c} \frac{x^{2}+x+1-x^{2}}{x^{2}+x+1}=3-\sum_{c y c} \frac{x^{2}}{x^{2}+x+1} \leq 2
$$


or

$$
\sum_{c y c} \frac{x^{2}}{x^{2}+x+1} \geq 1
$$

which is true from inequality (6).

4. Modifications of the original inequality. In this section we consider modifications of the original inequality (1) providing that $x y z=1$ for $x, y, z \geq 0$.

1. Inequality (1) is equivalent to

$$
\frac{x^{2}-1}{x^{3}-1}+\frac{y^{2}-1}{y^{3}-1}+\frac{z^{2}-1}{z^{3}-1} \leq 0 .
$$

This form leads to generalization with more powers, cf. below.

2. Inequality (1) is equivalent to

$$
\frac{x^{2}}{x^{2}-x+1}+\frac{y^{2}}{y^{2}-y+1}+\frac{z^{2}}{z^{2}-z+1} \leq 3 .
$$

3. Let us take $x \rightarrow \frac{1}{x}, y \rightarrow \frac{1}{y}, z \rightarrow \frac{1}{z}$. Then we derive another equivalent form of inequality (1)

$$
\frac{x-x^{2}}{x^{2}-x+1}+\frac{y-y^{2}}{y^{2}-y+1}+\frac{z-z^{2}}{z^{2}-z+1} \leq 0,
$$

due to the functional equation

$$
f\left(\frac{1}{x}\right)=-x f(x)
$$

for the function

$$
f(x)=\frac{x-1}{x^{2}-x+1} .
$$

So it seems possible to generalize the original inequality in terms of functional equations too.

Leads to one more change of variables $x \rightarrow x y, y \rightarrow y z, z \rightarrow x z$ :

$$
\frac{x y-1}{x^{2} y^{2}-x y+1}+\frac{y z-1}{y^{2} z^{2}-y z+1}+\frac{x z-1}{x^{2} z^{2}-x z+1} \leq 0
$$

or similarly to 18

$$
\frac{x y-x^{2} y^{2}}{x^{2} y^{2}-x y+1}+\frac{y z-y^{2} z^{2}}{y^{2} z^{2}-y z+1}+\frac{x z-x^{2} z^{2}}{x^{2} z^{2}-x z+1} \leq 0 .
$$


It is also possible to consider generalizations of (1) under the most general transformations

$$
x \rightarrow g(x, y, z), y \rightarrow h(x, y, z), z \rightarrow \frac{1}{g(x, y, z) h(x, y, z)}
$$

with positive functions $g(x, y, z), h(x, y, z)$ still preserving the condition $x y z=1$.

4. A number of cyclic inequalities follow from previous ones by substitution

$$
x=\frac{a}{b}, y=\frac{b}{c}, z=\frac{c}{a}, x y z=1 .
$$

On this way we derive from (1), (3)-(8) the following cyclic inequalities:

$$
\begin{aligned}
& \frac{a b-b^{2}}{a^{2}-a b+b^{2}}+\frac{b c-c^{2}}{b^{2}-b c+c^{2}}+\frac{c a-a^{2}}{c^{2}-c a+a^{2}} \leq 0, \\
& \frac{b^{2}}{a^{2}-a b+b^{2}}+\frac{c^{2}}{b^{2}-b c+c^{2}}+\frac{a^{2}}{c^{2}-c a+a^{2}} \leq 3, \\
& \frac{a b}{a^{2}-a b+b^{2}}+\frac{b c}{b^{2}-b c+c^{2}}+\frac{c a}{c^{2}-c a+a^{2}} \leq 3, \\
& \frac{a b-b^{2}}{a^{2}+a b+b^{2}}+\frac{b c-c^{2}}{b^{2}+b c+c^{2}}+\frac{c a-a^{2}}{c^{2}+c a+a^{2}} \leq 0, \\
& \frac{b^{2}}{a^{2}+a b+b^{2}}+\frac{c^{2}}{b^{2}+b c+c^{2}}+\frac{a^{2}}{c^{2}+c a+a^{2}} \geq 1, \\
& \frac{a b}{a^{2}+a b+b^{2}}+\frac{b c}{b^{2}+b c+c^{2}}+\frac{c a}{c^{2}+c a+a^{2}} \leq 1, \\
& \frac{a b+b^{2}}{a^{2}+a b+b^{2}}+\frac{b c+c^{2}}{b^{2}+b c+c^{2}}+\frac{c a+a^{2}}{c^{2}+c a+a^{2}} \leq 2 .
\end{aligned}
$$

Among cyclic inequalities ones of Schur, Nessbit and Shapiro are the most well-known cf. [1]-[3].

5. Some geometrical quantities connected with trigonometric functions and triangle geometry satisfy a condition $x y z=1$, cf. [4]-[6]. For example, we may use in standard notations for triangular geometry values:

$$
\begin{gathered}
x=\frac{a}{4 p}, y=\frac{b}{R}, z=\frac{c}{r} ; \\
x=\frac{a+b}{2}, y=\frac{b+c}{p}, z=\frac{a+c}{p^{2}+r^{2}+2 r R} ;
\end{gathered}
$$




$$
\begin{gathered}
x=R h_{a}, y=\frac{h_{b}}{2 p^{2}}, z=\frac{h_{c}}{r^{2}} ; \\
x=2 R^{2} \sin (\alpha), y=\frac{\sin (\beta)}{r}, z=\frac{\sin (\gamma)}{p} ; \\
x=\left(p^{2}-4 R^{2}-4 r R-r^{2}\right) \tan (\alpha), y=\frac{\tan (\beta)}{2 p}, z=\frac{\tan (\gamma)}{r} ; \\
x=\frac{\tan (\alpha)}{\tan (\alpha)+\tan (\beta)+\tan (\gamma)}, y=\frac{\tan (\beta)}{\tan (\alpha)+\tan (\beta)+\tan (\gamma)}, \\
x=\frac{\tan (\gamma)}{\tan (\alpha)+\tan (\beta)+\tan (\gamma)} ; \\
x=\frac{a}{4(p-a)}, y=\frac{b}{R(p-b)}, z=r \frac{\tan (\gamma / 2)}{p-c} \\
x=4 R \sin (\alpha / 2), y=\sin (\beta / 2), z=\frac{\sin (\gamma / 2)}{r} \\
x=4 R \cos (\alpha / 2), y=\cos (\beta / 2), z=\frac{\cos (\gamma / 2)}{p} .
\end{gathered}
$$

6. The above geometrical identities of the type $x y z=1$ which we use for applications of considered inequalities are mostly consequences of Vieta's formulas [5]. It is interesting to use these formulas for the cubic equation directly.

Theorem 2. Let $x, y, z$ be positive roots of the cubic equation with any real $a, b$

$$
t^{3}+a t^{2}+b t-1=0
$$

Then for these roots $x, y, z$ all inequalities of this paper are valid.

7. We can generalize inequalities $(3),(6)-(8)$ for more general powers. For this aim we use Bernoulli's inequalities [1]-[2]: for $u>0$ the following inequalities hold true

$$
\begin{gathered}
u^{\alpha}-\alpha u+\alpha-1 \geq 0,(\alpha>1 \text { or } \alpha<0), \\
u^{\alpha}-\alpha u+\alpha-1 \leq 0,(0<\alpha<1) .
\end{gathered}
$$


Lemma 2. Assume that $x, y, z$ are positive numbers such that $x y z=1$. Then the following inequality holds true:

$$
\left(\frac{1}{x^{2}-x+1}\right)^{\alpha}+\left(\frac{1}{y^{2}-y+1}\right)^{\alpha}+\left(\frac{1}{z^{2}-z+1}\right)^{\alpha} \leq 3
$$

for $0<\alpha<1$.

Proof. Let

$$
X=x^{2}-x+1, Y=y^{2}-y+1, Z=z^{2}-z+1 .
$$

Then we have

$$
\begin{gathered}
\left(\frac{1}{x^{2}-x+1}\right)^{\alpha}+\left(\frac{1}{y^{2}-y+1}\right)^{\alpha}+\left(\frac{1}{z^{2}-z+1}\right)^{\alpha} \leq \\
\leq \alpha\left(\frac{1}{X}+\frac{1}{Y}+\frac{1}{Z}\right)+3(1-\alpha) \leq 3 .
\end{gathered}
$$

Similarly we have from (7) that

$$
\left(\frac{x}{x^{2}+x+1}\right)^{\alpha}+\left(\frac{y}{y^{2}+y+1}\right)^{\alpha}+\left(\frac{z}{z^{2}+z+1}\right)^{\alpha} \leq 3-2 \alpha
$$

and from (8) we have

$$
\left(\frac{x+1}{x^{2}+x+1}\right)^{\alpha}+\left(\frac{y+1}{y^{2}+y+1}\right)^{\alpha}+\left(\frac{z+1}{z^{2}+z+1}\right)^{\alpha} \leq 3-\alpha .
$$

For $\alpha>1$ or $\alpha<0$ we have from $(6)$

$$
\left(\frac{1}{x^{2}+x+1}\right)^{\alpha}+\left(\frac{1}{y^{2}+y+1}\right)^{\alpha}+\left(\frac{1}{z^{2}+z+1}\right)^{\alpha} \geq 3-2 \alpha .
$$

5. Generalizations of original inequality to ones with a set of restrictions on symmetric functions. It is easy to show that the maximum of function (20) is attained for $x \geq 0$ at $x=2$ and equals $1 / 3$.

So the next unconditional inequality holds

$$
\sum_{k=1}^{k=n} \frac{x_{k}-1}{x_{k}^{2}-x_{k}+1} \leq \frac{n}{3} ; x_{k} \geq 0 .
$$




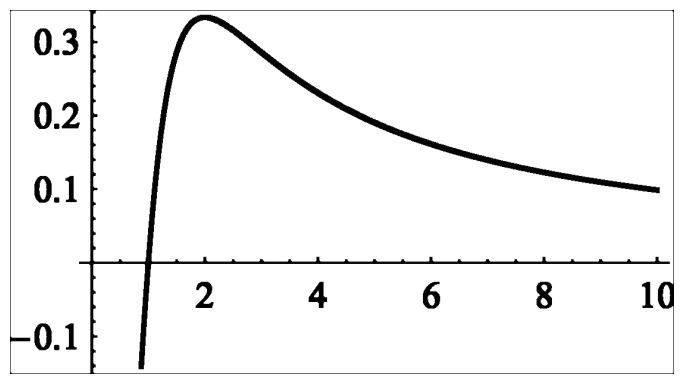

Figure 2: graph of function (20).

Consider symmetric functions

$$
S_{1}=\sum_{k=1}^{k=n} x_{k}, S_{2}=\sum_{k, m=1, k \neq m}^{n} x_{k} \cdot x_{m}, \ldots, S_{n}=x_{1} x_{2} \cdots x_{n} .
$$

\section{The generalized Damascus inequality (open problem).}

Prove the inequality

$$
\sum_{k=1}^{k=n} \frac{x_{k}-1}{x_{k}^{2}-x_{k}+1} \leq \frac{n}{3}-C\left(a_{1}, a_{2}, \ldots, a_{n}\right) ; x_{k} \geq 0,
$$

and find the best positive constant in it under conditions on symmetric functions

$$
S_{1}=a_{1}, S_{2}=a_{2}, \ldots, S_{n}=a_{n}
$$

with may be some restrictions in $(32)$ omitted.

The unconditional constant for positive numbers in (31) is $C=0$ and the original inequality gives $C=1$ in case $n=3$ and a single restriction $S_{3}=1$ in the list $(32)$.

It seems that the problem to find the sharp constant in inequality (31) under general conditions $(32)$ is a difficult problem.

For three numbers so more inequalities of type (31) may be considered, e.g.

1. Prove inequality (31) for positive numbers under condition $S_{1}=1$ and find the best constant for this case.

2. Prove inequality (31) for positive numbers under condition $S_{2}=1$ and find the best constant for this case.

Also combined conditions may be considered. 
3. Prove inequality (31) for positive numbers under conditions $S_{1}=a$, $S_{2}=b$ and find the best constant $C(a, b)$ in $(31)$ for this case.

6. Symmetricity of symmetric inequalities. There are many inequalities that are written in terms of symmetric functions as $F(p, q) \leq$ $\leq 0(\geq 0)$, where

$$
p=S_{1}=x+y+z, q=S_{2}=x y+y z+z x, r=S_{3}=x y z=1 .
$$

The following Lemma enlarges the amount of inequalities that one can obtain as a series of very complicated inequalities.

Lemma 3. If the inequality

$$
F(p, q) \leq 0(\geq 0)
$$

holds true, then the following inequalities are satisfied:

$$
\text { (i) } F(q, p) \leq 0(\geq 0) \text {, }
$$

and

$$
\text { (ii) } F\left(q^{2}-2 p, p^{2}-2 q\right) \leq 0(\geq 0) .
$$

Proof. (i). Assume that

$$
F(p, q)=F(x+y+z, x y+y z+z x) \geq 0 .
$$

Using transformations

$$
x \rightarrow x y, y \rightarrow y z, z \rightarrow z x
$$

we obtain

$$
\begin{gathered}
F(p, q)=F(x y+y z+z x, x y y z+y z z x+z x x y)= \\
\quad=F(x y+y z+z x, x+y+z)=F(q, p) \geq 0 .
\end{gathered}
$$

Notice that we can also use transformations

$$
x \rightarrow \frac{1}{x}, y \rightarrow \frac{1}{y}, z \rightarrow \frac{1}{z} .
$$

(ii). Now assume that

$$
F(p, q)=F(x+y+z, x y+y z+z x) \geq 0 .
$$


Using transformations

$$
x \rightarrow \frac{x y}{z}, y \rightarrow \frac{y z}{x}, z \rightarrow \frac{z x}{y}
$$

we derive

$$
\begin{gathered}
\frac{x y}{z}+\frac{y z}{x}+\frac{z x}{y}=x^{2} y^{2}+y^{2} z^{2}+z^{2} x^{2}= \\
=(x y+y z+z x)^{2}-2(x+y+z)=q^{2}-2 p .
\end{gathered}
$$

Also it follows

$$
\begin{gathered}
\frac{x y}{z} \frac{y z}{x}+\frac{y z}{x} \frac{z x}{y}+\frac{z x}{y} \frac{x y}{z}= \\
=\frac{y}{z x}+\frac{z}{x y}+\frac{x}{y z}=x^{2}+y^{2}+z^{2} \\
=(x+y+z)^{2}-2(x y+y z+z x)=p^{2}-2 q .
\end{gathered}
$$

The proof is complete.

At the end we propose an unsolved problem.

Open problem. Find all possible non-negative values of four variables $x_{1}, x_{2}, x_{3}, x_{4}$ with restriction $x_{1} \cdot x_{2} \cdot x_{3} \cdot x_{4}=1$ for which the next inequality holds

$$
\sum_{k=1}^{4} \frac{x_{k}-1}{x_{k}^{2}-x_{k}+1} \leq 0 .
$$

As we know from the example at the beginning of the paper the inequality (33) is not true for all such values, e.g. it fails for $x_{1}=x_{2}=x_{3}=2$, $x_{4}=1 / 8$.

Acknowledgment. The second author was partially supported by the grant of the Ministry of Education and Science of the Russian Federation (the Agreement number 02.a03.21.0008 of 24 June 2016). The authors are thankful to reviewers for useful suggestions and corrections.

\section{References}

[1] Mitrinović D. S., Pečarić J., Fink A. M. Classical and New Inequalities in Analysis. Springer, 1993.

[2] Mitrinović D. S., (in cooperation with Vasić P.M.). Analytic Inequalities. Springer, 1970. 
[3] Marshall A. W., Olkin I., Arnold B. C. Inequalities: Theory of Majorization and Its Applications. Second Edition. Springer, 2011.

[4] Mitrinović D. S., Pečarić J., Volenec V. Recent Advances in Geometric Inequalities. Kluwer, 1989.

[5] Soltman V. P., Meidman S. I. Equalities and Inequalities in a Triangle (in Russian). Kišinev, Štiinća Publishing, 1982.

[6] Bottema O., Djordjević R. Ž., Janić R. R., Mitrinović D. S., Vasić P. M. Geometric Inequalities. Groningen, 1969.

Received November 06, 2016.

In revised form, December 04, 2016.

Accepted December 05, 2016.

Department of Basic Sciences, Arab International University

P.O.Box 10409, Damascus, Syria

E-mail: fmdan@scs-net.org

Voronezh Institute of the Ministry of Internal Affairs of Russia

53, Patriotov pr., Voronezh, 394065, Russia;

Peoples' Friendship University of Russia

6, Miklukho-Maklaya st., Moscow, 117198, Russia

E-mail : pochtaname@gmail.com 